\title{
Correlative Transmission Electron Microscopy and Atom-Probe Tomography of an Iron Meteorite.
}

Surya S. Rout ${ }^{1,2}$, Philipp R. Heck ${ }^{1,2}$, Dieter Isheim ${ }^{4}$, Thomas Stephan ${ }^{2,3}$, Andrew M. Davis ${ }^{2,3}$, and David N. Seidman ${ }^{4}$.

${ }^{1}$ Robert A. Pritzker Center for Meteoritics and Polar Studies, The Field Museum of Natural History, 1400 S Lake Shore Drive, Chicago IL 60605, USA

${ }^{2}$ Chicago Center for Cosmochemistry, Chicago IL 60637, USA

${ }^{3}$ Department of Geophysical Sciences, The University of Chicago, 5734 S Ellis Avenue, Chicago IL 60637, USA

${ }^{4}$ Northwestern University Center for Atom-Probe Tomography, and Department of Materials Science \& Engineering, Northwestern University, 2220 Campus Drive, Evanston, IL 60208, USA

Correlative use of transmission electron microscopy (TEM) and atom-probe tomography (APT) provides complementary structural and chemical information with a unique combination of atomic-level spatial resolution and single-atom analytical sensitivity [1,2]. Correlated TEM/APT studies are state of the art in material science [3]. In our effort to broaden the application of APT in cosmochemistry [4], we expand our range of projects from meteoritic nanodiamonds [5] and presolar silicon carbide [6,7] to other samples. Here, we present new data from a correlated TEM/APT study of an iron meteorite [8].

Iron meteorites are $\mathrm{Fe}-\mathrm{Ni}$ alloys with minor amounts of $\mathrm{Co}, \mathrm{P}, \mathrm{S}$, and $\mathrm{C}$. They crystallized with extremely slow cooling rates $(0.2$ to $6000 \mathrm{~K} / \mathrm{Ma})$ within the metallic cores of asteroids during which the characteristic Widmanstätten pattern forms by nucleation and growth of Ni-poor kamacite $(\alpha-b c c$, ferrite) from taenite $\left(\gamma\right.$-fcc, austenite). The phase transformation occurring below $400^{\circ} \mathrm{C}$ involves a eutectoid reaction that results in formation of tetrataenite (FeNi) at the kamacite-taenite (K-T)_interface. The microstructure of the K-T interface and tetrataenite has been studied in detail using TEM [9]. One of our motivations is to take advantage of the superior spatial resolution and sensitivity of APT to study the composition of the K-T region on the nanoscale within fast cooled iron meteorites.

We prepared a polished section from the Bristol IVA iron meteorite (Field Museum specimen ME2248),. Bristol cooled at a relatively fast rate ( 250 K/Ma; [10]) and experienced comparably low shock pressures ( $<13 \mathrm{GPa}$; [10]). We used a Zeiss EVO 60 SEM equipped with an Oxford AZtec SDD EDS system to image the polished section. A Zeiss 1540 XB FIB-SEM was utilized to prepare sharp nanotips for APT from a $10 \times 5 \mu \mathrm{m}^{2}$ lamella perpendicular to the $\mathrm{K}-\mathrm{T}$ interface. The nanotips were attached to a copper half-grid with five presharpened posts. The grid was attached to a tomographic tip, fitted to a tomographic TEM holder (Hummingbird Scientific). TEM analysis was performed using a FEI Tecnai F20ST TEM prior to APT. APT was performed using a Cameca LEAP 4000XSi.

The TEM image of a tip (Fig. 1a) shows the presence of a clear interface, which was identified as the kamacite/taenite interface with EDS and electron diffraction. The 3-D tomographic reconstruction (Fig. 1b) shows isoconcentration surfaces for $\mathrm{Ni}$ and $\mathrm{Co}$; 3-D surfaces delineating regions with a concentration greater than a given threshold value for each element. The reconstruction shows clearly that Fe-rich (magenta dots) and Ni-rich (green) isoconcentration surface regions are separated by an interface, which represents the K-T interface, also seen in the TEM image (Fig. 1a). In a concentration profile obtained from the atom-probe data perpendicular to the $\mathrm{K}-\mathrm{T}$ interface, the $\mathrm{Fe}$ concentration 
decreases from $~ 95.6$ to $45.2 \mathrm{wt} \%$, and the Ni concentration increases from 5 to $54.7 \mathrm{wt} \%$. The high Ni concentrations of $54.7 \mathrm{wt} \%$ had not been detected before along a $\mathrm{K}-\mathrm{T}$ interface within a IVA iron meteorite. Co is concentrated in kamacite and decreases from $0.5 \mathrm{wt} \%$ in kamacite to $0.02-0.2 \mathrm{wt} \%$ in taenite. High Ni concentration was measured in the Santa Clara IVB iron meteorite using atom probe field ion microscopy [11] - a non-tomographic technique - and also within a mesosiderite $(65.5 \pm 3 \mathrm{wt} \%)$ using TEM [12]. We also observed variations in $\mathrm{Ni}$ and $\mathrm{Fe}$ concentrations within the tetrataenite suggesting the presence of inhomogeneities or nanostructures within it. The high Ni concentrations we measured in tetrataenite confirm predictions by the Fe-Ni phase diagram.
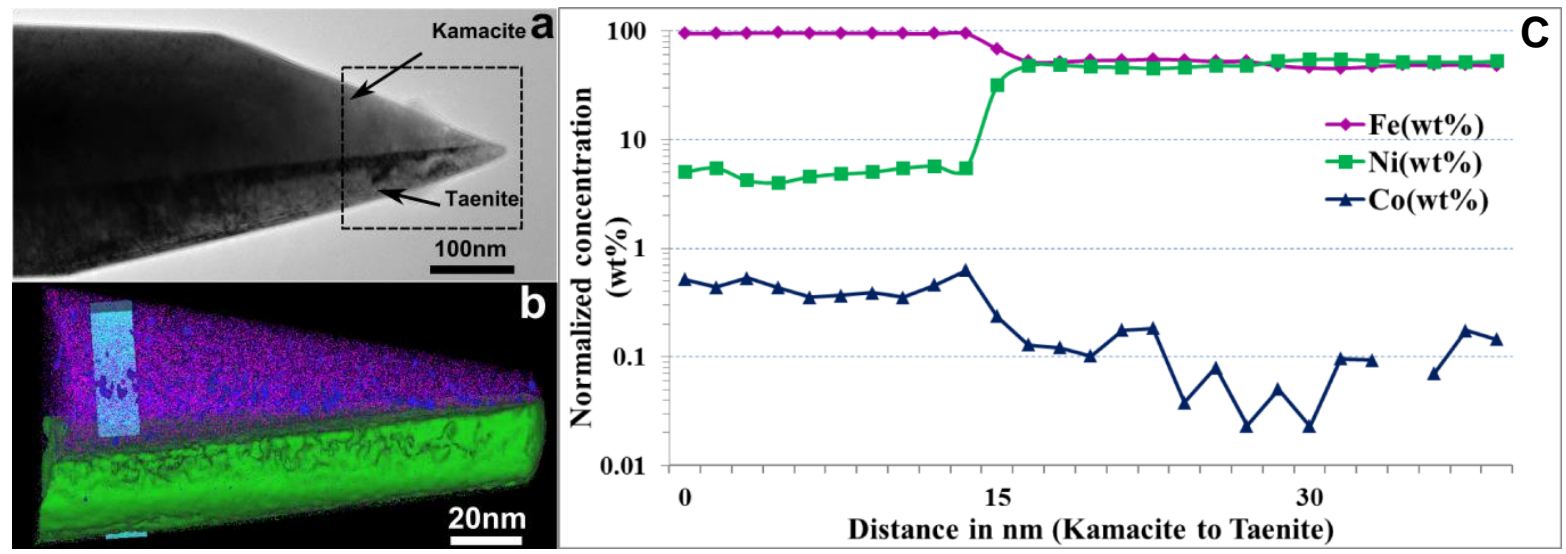

Figure 1. (a) TEM image of a nanotip prepared from the K-T interface. The region of APT reconstruction is shown with dashed lines. Corresponding APT reconstruction (b) with isoconcentration surfaces of $\mathrm{Ni}$ (green), and cobalt (dark blue) along with the distribution of Fe atoms (magenta dots) is shown. (c) Concentration profile along the long axis of the prism (cyan) shown in (b).

\section{References}

[1] Seidman D. N. and Stiller K. MRS Bulletin 34 (2009), p. 717-724.

[2] Kelly T. F. and Larson D. J. Annual Review of Material Science 42 (2012), p. 1-31.

[3] Moutanabbir O. et al, Nature 496 (2013), p. 78-82.

[4] Heck P. R. et al, 45 ${ }^{\text {th }}$ Lunar and Planetary Science Conference (2014), \#1811.

[5] Heck P. R. et al, Meteoritics \& Planetary Science 49 (2014), p. 453-467.

[6] Heck P. R. et al, $41^{\text {st }}$ Lunar and Planetary Science Conference (2010), \#2112.

[7] Stadermann F. J. et al, 41 ${ }^{\text {st }}$ Lunar and Planetary Science Conference (2010), \#2134.

[8] Rout S. S. et al, 46 ${ }^{\text {th }}$ Lunar and Planetary Science Conference (2015), \#2938.

[9] Goldstein J. I. et al, Chemie der Erde 69 (2009), p. 293-325.

[10] Goldstein J. I. et al, Meteoritics \& Planetary Science 44 (2009), p. 343-358.

[11] Miller M. K. and Russell K. F. Surface Science 226 (1992), p. 441-445.

[12] Reuter K. B. et al, Geochimica et Cosmochimica Acta 61 (1988), p. 2943-2956.

[13] Acknowledgements: We thank D. Miller and N. Zaluzec for their help with FIB and TEM at the Electron Microscopy Center, Argonne National Laboratory. Use of the Center for Nanoscale Materials was supported by the U. S. Department of Energy, Office of Science, Office of Basic Energy Sciences, under Contract No. DE-AC02-06CH11357. LEAP was done at NUCAPT, a shared facility at the Materials Research Center of Northwestern University. The LEAP was purchased and upgraded with funding from NSF-MRI and ONR-DURIP. PRH acknowledges funding from the Tawani Foundation and from W. H. Ganz III. 